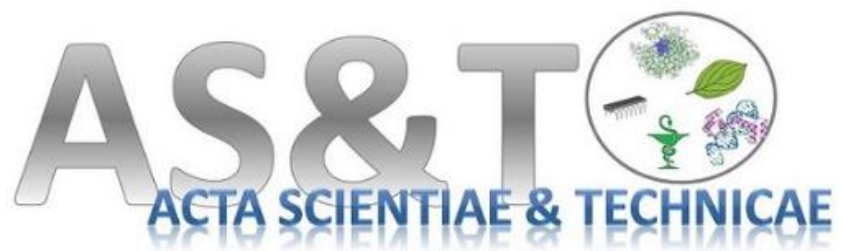

\section{Influências de preparação da superfície nos resultados de análise das tensões mecânicas por difração de raios-X}

\author{
Heittor Werner da Silva ${ }^{1}$, José William de Lana ${ }^{1}$,Tetyana Gurova ${ }^{1}$
}

\section{AUTHOR AFILIATIONS}

1 - Departamento da Construção Naval, Laboratório de Ensino e Pesquisa da Construção Naval/ UEZO, Av Manuel Caldeira de Alvarenga, 1203, Campo Grande. 23040-200 - Rio de Janeiro, RJ Brasil

\section{CONTACT}

gurova@lts.coppe.ufrj.br

\begin{abstract}
Residual stresses are the result of a set of forces that are present in a metal material independent of external actions, and these stresses can directly affect material's life, especially if new stresses are introduced into the material through surface treatments. Therefore, this production will analyze by X-ray diffraction method the influence of surface preparation on the residual stresses of a steel piece.
\end{abstract}

Keywords: Surface Treatments, Residual Stress, X-Ray Diffraction

\section{RESUMO}

Tensões residuais são o resultado de um conjunto de forças que estão presentes em um material metálico independente de ações externas, e essas tensões podem afetar diretamente a vida do material, especialmente se novas tensões forem introduzidas no material por meio de tratamentos de superfície. Portanto, esta produção analisará pelo método de difração de raios X a influência do preparo da superfície sobre as tensões residuais de uma peça de aço.

Palavras-chave: Tratamentos de superfície, estresse residual, difração de raios$\mathrm{X}$ 


\section{INTRODUÇÃO}

Determinados materiais fazem parte dos principais segmentos da economia moderna. No ramo naval o aço é base para quase todos os equipamentos e maquinário, bem como na construção da embarcação em si, estando presentes em elementos estruturais, chapas, revestimentos, acabamentos e elementos de fixação. O conhecimento das propriedades destes materiais é importante para definir o melhor aproveitamento de acordo com sua finalidade.

Um dos grandes fatores que influenciam na vida útil de determinados materiais é a tensão residual que pode ser definida com qualquer tensão existente em determinado material ao longo de seu volume, independe da existência de carga externa e que se encontra presente no material a partir da sua fabricação. A tensão residual é importantíssima para a definição das características mecânicas dos materiais que por sua vez permite o planejamento de manutenção ou reparo na peça analisada. O conhecimento da tensão residual permite que sejam feitos alterações e tratamentos no material visando o melhoramento de suas propriedades, prolongando sua vida útil e diminuindo a possibilidade do surgimento de trincas.

As tensões residuais podem ser originadas através de vários processos desde a fabricação do material, mecanismos referentes ao processo em si, até os tratamentos finais, como descrito por Ruud (1992) e com isso essas tensões podem ser classificadas de acordo com sua origem, sejam elas térmicas, químicas ou mecânicas. Essas tensões possuem um valor máximo em módulo denominado limite de escoamento do material e conjuminadas a fatores externos, pode exceder esse limite colapso do mesmo material em questão (DO CARMO, 2006).

Existem vários processos tecnológicos que podem ser aplicados no material para minimizar a implicação da tensão residual em sua vida útil, entre eles, tratamentos térmicos e tratamentos mecânicos, como por exemplo, shot-peening.

O polimento mecânico de metais é fundamental no processo de tratamento da superfície do material, ele geralmente é utilizado com a finalidade de aumentar o desempenho do 
material, corrigir erros como riscos, buracos, rebarbas e imperfeições no material e melhorar esteticamente a aparência da peça. $\mathrm{O}$ polimento mecânico pode ser feito de forma manual onde a pessoa utiliza lixas e panos para passar sobre a superfície da peça e o mecânico que necessita de uma máquina para movimentar as lixas e panos e gerar o atrito com a peça.

Sabe-se que o polimento mecânico altera as tensões residuais que estão agindo sobre o material. Neste trabalho busca-se saber o quanto e como as tensões variaram no material antes e depois do polimento mecânico.

\section{MÉTODOS}

Para os ensaios foram utilizadas amostras de tamanho $70 \times 35 \times 6$ de aço naval DH36 formado com ligas de manganês e silício, possuindo densidade média $7,8 \mathrm{~g} / \mathrm{cm}^{3}$. O limite de escoamento dos materiais em geral que é o ponto onde começa a deformação irrecuperável do material para esse material o limite de escoamento é em torno de 355MPa. Para medir tensões residuais da amostra no estado inicial, no local de medição foi aplicado polimento eletrolítico.

Primeiramente amostra foi submetida ao lixamento manual com lixa grau 120 , onde o movimento foi executado apenas na direção longitudinal da amostra (na direção do seu lado mais comprido). $\mathrm{Na}$ segunda etapa, a mesma amostra foi submetida ao lixamento manual com movimento apenas na direção transversal e lixa grau 120. Na terceira etapa foi realizado polimento da amostra com uma lixadeira politriz motorizada com rotação variável entre 300 e 600 rpm, usando lixas d'água de 80, 120, 240, 360, $500,600,800,1200,1500,1800$ e por fim 2000 para então ser utilizada a pasta de diamante de $3 \mu \mathrm{m}$. Foi importante a adição de água enquanto a peça estava sobre o processo de polimento para evitar danos ao material pelo aquecimento gerado no atrito entre o metal e a lixa.

Os valores absolutos das tensões residuais serão medidas com equipamento portátil de raiosX RAYSTRESS, Figura 1, que utiliza o método de dupla exposição e radiação $\mathrm{K}_{\alpha}$ do $\mathrm{Cr}$. 


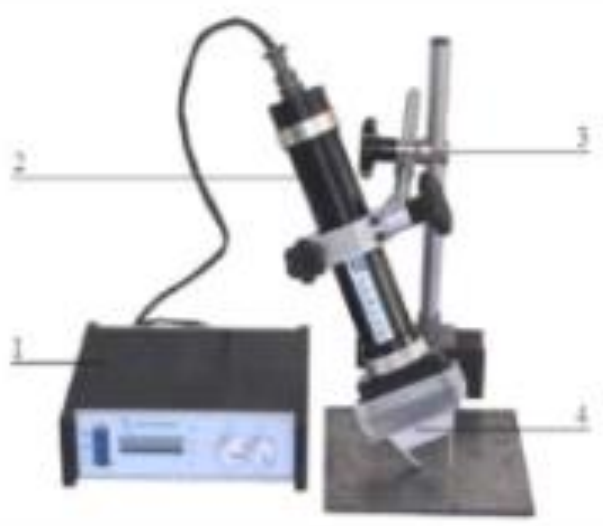

Figura 1. Equipamento portátil RAYSTRESS

As seguintes partes são incluídas como componentes deste equipamento, (GUROVA et al. 2017): (1) - Unidade de controle com fonte de alta tensão que permite monitoramento e o ajuste do nível de potência de alimentação do tubo de raios-X; (2) - Fonte de alta tensão e tubo de raios$\mathrm{X}$. Uma das qualidades do equipamento é que o tubo de raios-X é acoplado à fonte de alta tensão. A tensão e a corrente de trabalho da fonte de raios-X é de $25 \mathrm{kV}$ e 1,5 mA, respectivamente. O tubo de raios-X possui dois anodos de cromo com resfriamento ao ar, e produz dois feixes convergentes de raios-X para realizar a técnica de duas exposições de medição de tensões por raiosX. O ângulo de convergência dos feixes de raios$\mathrm{X}$ é de $50^{\circ}$; (3) - O suporte magnético permite instalar o equipamento diretamente na peça analisada e ajustar o equipamento na posição de exposição; (4) - O colimador com cassete para filme de raios-X. Duas janelas no cassete permitem captar partes das linhas difratadas no intervalo angular de $2 \theta$ de $148^{\circ}$ a $164^{\circ}$.

Para obter os valores principais de tensão e, consequentemente, os valores de tensão de Von Mises é necessário efetuar medidas de tensão ao longo de três direções. No caso elástico o estado de tensão representado pelo tensor simétrico de segunda ordem T. Para qualquer direção $\bar{n}$, a tensão resultante é obtida como ${ }^{\bar{\sigma}=T \bar{n}}$ e pode ser decomposto em componente normal $\sigma_{n}$ e componentes tangenciais $\sigma_{z}$. A técnica de difração de raio-X permite encontrar a componente normal da tensão na direção ${ }^{\bar{n}}$. A distribuição de tensão superficial é considerada como um estado de tensão no plano. Introduzindo um sistema ortogonal (XOY), o valor da tensão pode ser expresso na forma matricial:

$$
\left[\begin{array}{ll}
\sigma_{x} & \tau_{x y} \\
\tau_{x y} & \sigma_{y}
\end{array}\right]
$$


Assim, o estado de tensão do plano no

ponto é descrito por três componentes $\sigma_{x}, \sigma_{y} \mathrm{e}$ $\tau_{x y}$. Seja $\sigma_{0} \mathrm{e}^{\sigma_{90}}$ os valores medidos das componentes normais da tensão em duas direções ortogonais $0^{\circ}$ e $90^{\circ}$, correspondendo ao eixo $\mathrm{OX}$ e OY. Então $\sigma_{x}=\sigma_{0}, \sigma_{y}=\sigma_{90}$ e a componente normal da tensão em qualquer direção arbitrária $\alpha$ em relação ao eixo OX é definido como:

$\sigma_{\alpha}=\frac{\sigma_{0}+\sigma_{90}}{2}+\frac{\sigma_{0}-\sigma_{90}}{2} \cos 2 \alpha+\tau_{0,90} \sin 2 \alpha$

$\tau_{0,90}=\sigma_{45}-0.5\left(\sigma_{0}+\sigma_{90}\right)$

Então, a tensão principal $\sigma_{1}, \sigma_{\mathrm{II}}$, a tensão cisalhante máxima ${ }^{\tau_{\max }}$ ) e a tensão efetiva de $\operatorname{von} \operatorname{Mises}\left({ }^{\sigma_{\text {eff }}}\right)$ são obtidas como:

$$
\sigma_{i}=\frac{\sigma_{0}+\sigma_{90}}{2}+\sqrt{\left(\frac{\sigma_{0}-\sigma_{90}}{2}\right)^{2}+\tau_{0,90}^{2}}
$$

$$
\sigma_{l /}=\frac{\sigma_{0}+\sigma_{90}}{2}-\sqrt{\left(\frac{\sigma_{0}-\sigma_{90}}{2}\right)^{2}+\tau_{0,90}^{2}},
$$

$$
\tau_{\max }=\sqrt{\left(\frac{\sigma_{0}-\sigma_{90}}{2}\right)^{2}+\tau_{0,90}^{2}}
$$

$$
\sigma_{e f f}=\sqrt{\sigma_{l}^{2}+\sigma_{I I}^{2}-\sigma_{l} \sigma_{l l}} .
$$

\section{RESULTADOS}

Resultados das medições de tensões estão apresentados na Tabela 1. Anotações utilizadas: $\bar{n}$ - direção de medição da tensão, L - direção longitudinal a amostra; $\mathrm{T}$ - direção transversal a amostra; D - direção com ângulo $45^{\circ}$ entre as duas primeiras; $\sigma_{\mathrm{n}}$ - valore da componente normal da tensão medida na direção $\mathrm{n} ; \sigma_{l}$ e $\sigma_{l l}$ - valores calculados das tensões principais; $\sigma_{e f f}$ - tensão calculada de von Mises. Com número $\mathrm{N}=1$ são marcadas as medições das tensões na amostra 
após o polimento eletrolítico; número $\mathrm{N}=2$ corresponde a condição após lixamento apenas na direção longitudinal; número $\mathrm{N}=3$ - lixamento na direção longitudinal seguida por lixamento na direção transversal; número $\mathrm{N}=4$ - após polimento na politriz.

Tabela 1. Valores das tensões residuais, $\mathrm{MPa}$

\begin{tabular}{|c|c|c|c|c|c|}
\hline $\mathrm{N}$ & $\bar{n}$ & $\sigma_{\mathrm{n}}$ & $\sigma_{l}$ & $\sigma_{l l}$ & $\sigma_{e f f}$ \\
\hline \multirow{3}{*}{1} & $\mathrm{~L}$ & +122 & \multirow{3}{*}{+193} & \multirow{3}{*}{+116} & \multirow{3}{*}{169} \\
\hline & D & +176 & & & \\
\hline & $\mathrm{T}$ & +187 & & & \\
\hline \multirow{3}{*}{2} & $\mathrm{~L}$ & -320 & \multirow{3}{*}{-114} & \multirow{3}{*}{-329} & \multirow{3}{*}{289} \\
\hline & D & -263 & & & \\
\hline & $\mathrm{T}$ & -122 & & & \\
\hline \multirow{4}{*}{3} & $\mathrm{~L}$ & -151 & \multirow{3}{*}{-150} & \multirow{3}{*}{-271} & \multirow{3}{*}{235} \\
\hline & D & -202 & & & \\
\hline & $\mathrm{T}$ & -270 & & & \\
\hline & $\mathrm{L}$ & -130 & & & \\
\hline
\end{tabular}

\begin{tabular}{|l|l|l|l|l|l|}
4 & $\mathrm{D}$ & -147 & -125 & -149 & 139 \\
\cline { 1 - 3 } & $\mathrm{T}$ & -144 & & & \\
\hline
\end{tabular}

Resultados de medição das tensões na amostra após polimento eletrolítico apresenta estado real das tensões residuais da amostra. As tensões são trativas e os valores medidos nas direções longitudinal e transversal ficam próximos aos valores principais.

Lixamento manual da amostra apenas na direção longitudinal com lixa grau 120 introduz tensões residuais de compressão na amostra. As direções longitudinal e transversal ficam próximos das direções principais das tensões residuais. Não foi observado o surgimento das tensões trativas na direção ortogonal (transversal) à direção do lixamento, como é afirmado nos trabalhos (PINTO, 2017; CARVALHO et al. 2018).

Lixamento manual da amostra na direção longitudinal seguida do lixamento na direção transversal com lixa grau 120 introduz tensões residuais de compressão na amostra. As direções 
longitudinal e transversal de novo são direções

principais das tensões resíduas, porem apresentam distribuição mais uniforme comparando com lixamento apenas na direção longitudinal.

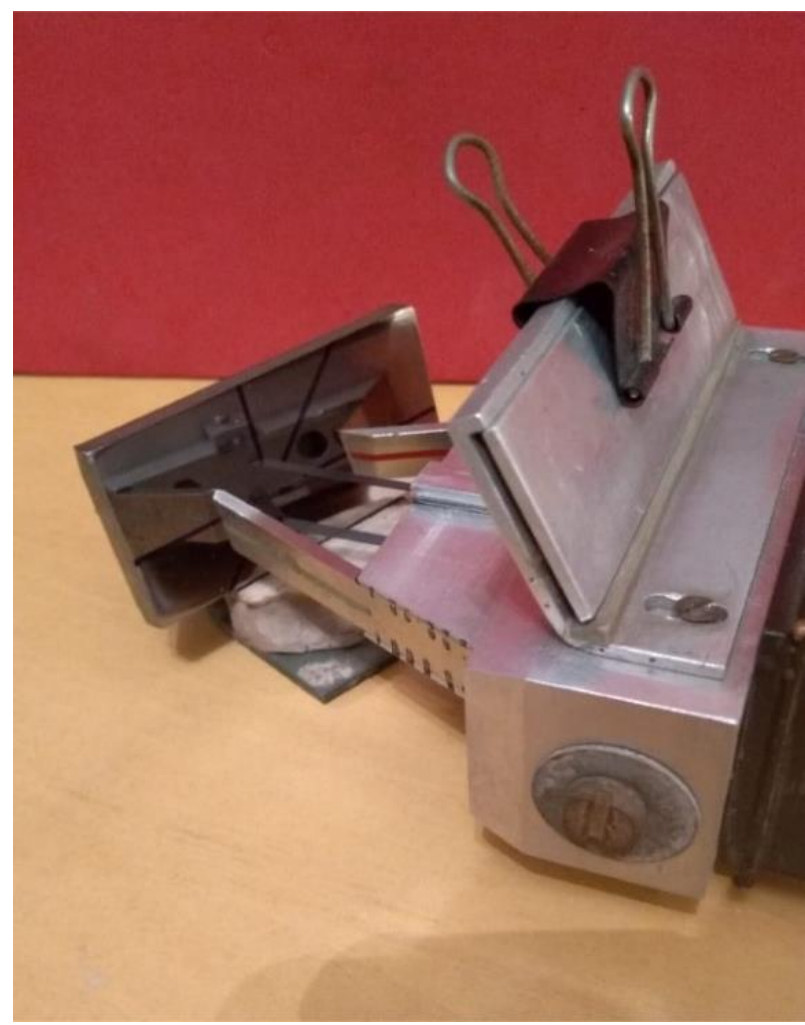

Figura 2. Medição das tensões na direção longitudinal da amostra após polimento na politriz.

Polimento mecânico na politriz: as tensões residuais continuam sendo tensões compressivas, porem ficaram distribuídas muito mais uniforme do que no caso do lixamento manual e a direção principal corresponde a direção diagonal da amostra, figura 2.
Observa-se que durante polimento eletrolítico foi removida uma camada superficial do material de aproximadamente $0.2 \mathrm{~mm}$. Camada de profundidade compatível com este valor foi removida durante polimento mecânico na politriz.

Porém, o polimento mecânico assim como lixamento manual mudam drasticamente o estado inicial das tensões residuais na amostra, medidos após polimento eletrolítico, passando do estado com tensões trativas para o estado com tensões compressivas. Exatamente por este motivo métodos mecânicos, como polimento, usinagem ou o uso de uma escova de aço devem ser evitados na preparação da amostra (FITZPATRICK et al. 2005), pois eles introduzirão tensões residuais superficiais adicionais na amostra a ser analisada e os resultados destas medições levam as conclusões equivocas sobre estado real das tensões (CEGLIAS, 2012).

\section{CONCLUSÕES}

O lixamento manual e o polimento mecânico introduzem tensões residuais de compressão na superfície da peça. É possível 
notar a não homogeneidade dos valores de tensões residuais quando realizado o lixamento manual, todavia quando utilizado a politriz para o polimento, é observado a distribuição mais homogênea dos valores das tensões na superfície da amostra analisada. Comparando os valores de von Mises das tensões induzidas durante lixamento manual e o polimento com politriz podemos observar que o procedimento de polimento introduz as tensões menores de compressão. Porém, o polimento assim como lixamento manual mudam drasticamente o estado inicial das tensões residuais na amostra, passando do estado com tensões trativas para o estado com tensões compressivas, sendo assim, os métodos mecânicos, como polimento, usinagem ou o uso de uma escova de aço devem ser evitados na preparação da amostra para medição das tensões por difração de raios-X. O único método de preparação fica limitado ao polimento eletrolítico.

\section{AGRADECIMENTOS}

Os autores agradecem a Fundação de Amparo à Pesquisa do Estado do Rio de Janeiro FAPERJ pelo auxílio a pesquisa (Gurova T.) e a empresa GURTEQ - Ensaios Não Destrutivos Ltda pelo auxílio operacional na medição das tensões.

\section{REFERÊNCIAS}

CARVALHO FILHO, E.T., MEDEIROS, J.T.N., MARTINEZ, L.G. and PINTO, V.C. Study of error analysis and sources of uncertainty in the measurement of residual stresses by the X-ray diffraction. Materials Research Proceedings, 6 (2018) 75-80.

CEGLIAS, R. B. Análise de tensão residual em tubo de aço API 5L X70. Dissertação de Mestrado, Instituto Militar de Engenharia, Rio de Janeiro, 2012, 111 p.

DO CARMO, D.C. Análise de tensão por ultrasom em uma barra de aço estrutural sob flexão. Dissertação de Mestrado, PEMM/COPPE/UFRJ, Rio de Janeiro, 2006.

FITZPATRICK, M.E., FRY, A.T., HOLDWAY, P., KANDIL, F.A., SHACKLETON, J. and SUOMINEN, L. Determination of residual stresses by X-ray diffraction - Issue 2 . Measurement good practice guide, No. 52, National Physical Laboratory, United Kingdom, 2005, 77 p.

GUROVA, T., ESTEFEN, S.F., LEONTIEV, A., BARBOSA, P.T., DE OLIVEIRA, F.A.L. Timedependent redistribution behavior of residual 
stress after repair welding. Welding in the World, 61 (2017) 507-515.

PINTO, V.C. Influência dos parâmetros de corte na integridade superficial do aço SAE 1045 após torneamento. Dissertação de Mestrado,
Universidade Federal do Rio Grande do Norte, Natal, 2017, 114 p.

RUUD, C.O. Residual stress and their measurement. $1^{\text {th }}$ International Conference on Quenching \& Control of Distortion, USA, 1992. 